\title{
Aufwärtskompatibel? Zur Bedeutung struktureller und doktrinärer Nationalismen für die digitale Konstellation
}

\author{
Wolf J. Schünemann iD
}

Angenommen: 2. August 2021 / Online publiziert: 30. August 2021

(C) Der/die Autor(en) 2021

Zusammenfassung Der Beitrag unternimmt den Versuch, theoretische Ansätze der Nationalismusforschung auf die digitale Konstellation zu übertragen. Lassen sich konstitutive Merkmale der digitalen Konstellation und aktuelle digitalpolitische Entwicklungen unter Rückgriff auf Nationalismustheorien erklären? Erweisen sich Nationalismen, denen eine Hauptfunktion bei der Integration prädigitaler Sozialräume und dem Aufbau einer politischen Weltordnung zugekommen ist, damit gleichsam als aufwärtskompatibel? Um die Beharrungskraft (nicht die Ursprünge) des Nationalen in der digitalen Konstellation zu erklären, stützt sich der Beitrag zunächst auf Ansätze konstruktivistischer Nationalismustheorie. Den Grundstein des Modells bilden strukturelle Nationalismen, verstanden als unideologische, unbewusste und vielfach unauffällige Denk- und Reproduktionsweisen des Nationalen. Diese bieten Anknüpfungspunkte für doktrinäre Nationalismen, die die Autonomie nationaler Gesellschaften thematisieren und ihre Bewahrung zur politischen Zielsetzung erheben. Aus der Kombination lassen sich aktuelle digitalpolitische Entwicklungen erklären. Zwecks empirischer Illustration der verschränkten Mechanismen (strukturell und doktrinär) fokussiert der Beitrag nacheinander auf drei digitalpolitische Handlungs- und Problemfelder: die Verwaltung kritischer Ressourcen am Beispiel des Domain-Name-Systems (DNS), die Cybersicherheitspolitik im Hinblick auf den Schutz kritischer Infrastrukturen sowie schließlich die Bekämpfung von Desinformationskampagnen durch die Regulierung von Kommunikationsströmen.

Schlüsselwörter Digitalpolitik · Nationalismus · Konstruktivismus ·

Cybersicherheit · Desinformation

Wolf J. Schünemann ( $₫)$

Institut für Sozialwissenschaften, Universität Hildesheim, Hildesheim, Deutschland

E-Mail: wolf.schuenemann@uni-hildesheim.de 


\title{
Upward compatible? On the relevance of structural and programmatic nationalisms for the governance of digitalization
}

\begin{abstract}
The article presents an attempt to apply theories of nationalism to the digital constellation. Can constitutive features of the digital constellation and current political conflicts on the governance of digitalization be explained based on nationalism theory? Do nationalisms having had a core function in integrating pre-digital societies and in building a political world order qualify as upward compatible? In order to explain the persistence (not the origins) of national thinking and practices in the digital constellation, the paper builds upon constructivist theories of nationalism. Structural nationalisms, understood as the non-ideological, unconscious and often unobstrusive ways of thinking and reproducing the nation, constitute the cornerstone of the combined approach. They can serve as connection points for programmatic forms of nationalism that emphasize the autonomy of national societies and transform it into political objectives. Combinations of structural and programmatic forms of nationalism help to understand current conflicts in digital politics. For the empirical illustration of combined mechanisms, the paper discusses three spotlight cases from the field of internet governance: the administration of critical resources with a focus on the Domain-Name-System (DNS), cybersecurity policy with a focus on critical infrastructures and finally the fight against disinformation campaigns with a focus on online content regulation.
\end{abstract}

Keywords Digital governance - Nationalism · Constructivism · Cybersecurity · Disinformation

\section{Einleitung}

Zu den eingeübten politischen Narrativen der Digitalisierung zählt ihre Erzählung im Sinne einer ,postnationale[n] Konstellation“ (Habermas 1998a). Das Narrativ von einer Überwindung nationalstaatlicher Ordnung nicht nur, aber auch durch Internet und Digitalisierung ergibt sich nicht allein aus frühen libertären Visionen für vernetzte Gesellschaften (Barlow 1996; Johnson und Post 1996) oder abgeleitet aus einflussreichen netzwerktheoretischen Arbeiten (Benkler 2006; Castells 2001), sondern es findet in den realen Entwicklungen technischer Infrastruktur, staatsferner Governance und transnationaler Datenströme auf den ersten Blick reichlich empirische Nahrung. Allerdings sind die Trends digitalisierungsinduzierter oder auf sie bezogener Globalisierung nicht ungebrochen. Gerade mit Blick auf Gegenwart und Zukunft des Internets werden unter den Schlagwörtern der „Balkanisierung“, der ,Fragmentierung“ oder des ,Splinternet“ seit geraumer Zeit gegenläufige Entwicklungen diskutiert, die ex negativo, nämlich ausgehend von der transnationalen Ambition, das Beharrungsvermögen nationalstaatlicher Ordnung thematisieren (Drake et al. 2016; Hill 2012; kritisch: Mueller 2017). Dies geschieht jedoch vielfach ohne die systematische Anknüpfung an sozialwissenschaftliche Theorieangebote. Dieser Theoriemangel wird aktuell insofern akuter, als in der Digitalpolitik eine teils offene Reorientierung an nationalen Mustern erkennbar wird. Als Illustrationen 
aus der jüngsten Vergangenheit sei nur auf die weithin und wiederkehrend geführten Debatten über digitale oder technologische Souveränität, zuletzt etwa im Zusammenhang mit dem 5G-Netzausbau (Pohle und Thiel 2020), auf nationale Maßnahmen zur Regulierung von Internetinhalten oder das zwischenstaatliche Konfliktgeschehen im Rahmen des so genannten Cyberkriegs hingewiesen. Diese Entwicklungen sind durch digitalisierungsinduzierte Transformationen allein nicht erklärbar und bedürfen einer breiteren gesellschafts- und politiktheoretischen Betrachtung. Dies leistet der vorliegende Beitrag aus Perspektive der Nationalismustheorie. Neben das in der politischen Debatte verbreitete Konzept der Souveränität ist jüngst auch der kritischere Term des Nationalismus (,data nationalism“, Chander und Lê 2015; ,techno-nationalism“, Möllers 2021) getreten. Aus politikwissenschaftlicher Perspektive stellt sich die Frage nach den Beiträgen von Nationalismustheorien zur Erklärung der digitalen Konstellation damit umso drängender.

Der vorliegende Beitrag unternimmt also den Versuch, die Theorie vom Nationalismus als gesellschafts- und politiktheoretisches Angebot der Moderne (Gellner 1999; Kedourie 1971; Kohn 1955) auf die digitale Konstellation zu übertragen. Lassen sich konstitutive Merkmale der digitalen Konstellation und aktuelle digitalpolitische Entwicklungen unter Rückgriff auf Nationalismustheorien erklären? Erweisen sich Nationalismen, denen eine Hauptfunktion bei der Integration prädigitaler Sozialräume und dem Aufbau einer politischen Weltordnung zugekommen ist, damit gleichsam als aufwärtskompatibel? Mit dem Versuch, die Beharrungskraft (und nicht die Ursprünge) des Nationalen in der digitalen Konstellation zu erklären, knüpft der Beitrag in erster Linie an jüngere konstruktivistische Ansätze an (Anderson 2006; Hobsbawm 2005). Den Grundstein des Modells bilden sog. strukturelle Nationalismen, verstanden als unideologische, unbewusste und vielfach unauffällige Denkund Reproduktionsweisen des Nationalen (s. auch Werron 2018). Ausgehend von dieser wissenssoziologischen Konzeption, stelle ich zudem eine Verbindung zu einer genuin politiktheoretischen Dimension her, indem ich Anknüpfungspunkte sog. doktrinärer Nationalismen identifiziere und auch diese zur Erklärung digitalpolitischer Entwicklungen vorschlage. Zwecks empirischer Illustration der verschränkten Mechanismen (strukturell und doktrinär) fokussiere ich nacheinander auf drei digitalpolitische Handlungs- und Problemfelder: die Verwaltung kritischer Ressourcen am Beispiel des Domain-Name-Systems (DNS), die Cybersicherheitspolitik im Hinblick auf den Schutz kritischer Infrastrukturen sowie schließlich die Bekämpfung von Desinformationskampagnen durch die Regulierung von Kommunikation.

\section{Transnationale Vision des Cyberspace versus nationalstaatliche Fragmentierung - Forschungsstand}

Für die theoretischen Entwürfe der digitalen Konstellation in Politikwissenschaft und angrenzenden Disziplinen ist der Nationalstaat auf den ersten Blick nicht prägend. Sowohl im Hinblick auf seine gesellschaftliche Integrationsleistung als auch seine politische Ordnungsfunktion haben Internet und Digitalisierung nach allgemeiner Ansicht dessen Relativierung beschleunigt, enstehen doch durch die globale Vernetzung alternative Kommunikations- und Sozialräume, welche nicht auf raum- 
zeitliche Kopräsenz im materiellen Raum angewiesen sind, um eine soziale Umwelt im Schütz'schen Sinn zu konstituieren (Schütz 1993; Pries 2010). ${ }^{1}$ Die Digitalisierung hat Globalisierungsprozesse beschleunigt und substantiell vertieft. Die partielle Erosion nationaler Identitäten kann eine Folge sein (Fukuyama 2018, S. 180ff.).

Auch die politischen Gestaltungsmöglichkeiten von Nationalstaaten werden in der digitalen Konstellation als herausgefordert angesehen: ,(state) sovereignty, traditionally thought to be the bedrock of modern politics, has become a contested concept" (Pohle und Thiel 2020, S. 7, s. auch den Beitrag von Neyer in diesem Sonderheft). Die funktionalen Sachzwänge transnationaler Interdependenzen, dominante marktliberale Paradigmen und innovative Governance-Formate jenseits von Staatlichkeit haben die Regulierung von Internet und Digitalisierung in den zurückliegenden Jahrzehnten geprägt (DeNardis 2014; Thiel 2019, S. 47). Insbesondere die Staaten des demokratischen Westens haben sich unter dem Eindruck eines liberalen Konsenses - zumindest bis in die jüngste Zeit - medienpolitische Zurückhaltung auferlegt (Vick 2001). Auch losgelöst von libertären Visionen für den Cyberspace (Barlow 1996; Johnson und Post 1996), scheinen nationalstaatliche Handlungsspielräume gegenüber neuen Internetökosystemen zunehmend begrenzt (s. auch Plattformgesellschaft, van Dijck et al. 2018).

Normative oder empirische Staatsferne sind allerdings zu keinem Zeitpunkt ohne Kontestation geblieben, sei es durch rivalisierende Allianzen in aufkeimenden geopolitischen Konflikten um die Regulierung des Internets (DeNardis 2014; Glen 2014), sei es in der wissenschaftlichen Debatte. Hier stellten als Skeptiker früher Stunde die Rechtswissenschaftler Jack Goldsmith und Tim Wu einen entschiedenen Gegenentwurf vor, indem sie Autoritätskonflikte über die Regulierung von Internetinhalten zwischen emergenten Plattformbetreibern und nationalen Jurisdiktionen diskutierten. Im Vorwort zu ihrem Buch „Who controls the Internet? Illusions of a Borderless World" wird gerade die unterschätzte Beharrlichkeit des Nationalstaats zur Ausgangsbeobachtung erklärt:

The Internet age is characterized by the incessant search for the newest ,new thing“. Our story, by contrast, is about old things - the enduring relevance of territory and physical coercion within nations, and cooperation and conflict between them. Territorial government is a persistent fact of human history that accommodates humanity in its diversity and allows it to flourish. Behind the mists and magic of the Internet lies an older and stronger order whose relevance remains inescapable. (Goldsmith und Wu 2006, S. xiii; s. auch Wu 1997)

Es ist unschwer zu erkennen, dass die Autoren dem Nationalstaat eine historische Tradition zuschreiben, die in der Geschichtswissenschaft mindestens umstritten ist (Hobsbawm 2005) und aus historischer Perspektive als Gegenposition zu transnationalen Visionen nicht plausibel erscheint. Dennoch prägt das territorialstaatliche Modell insbesondere die rechtswissenschaftliche Diskussion und hat angesichts aufbrechender Ordnungskonflikte bei der Governance von Digitalisierung die Verbrei-

\footnotetext{
1 Dabei darf allerdings nicht vergessen werden, dass schon der Nationalismus und überhaupt die Sozialkonzeptionen der Moderne die konkreten Gemeinschaftskontexte zugunsten abstrakterer Gesellschaftsbezüge hinter sich gelassen hatten (Habermas 1998b; Tönnies 1887).
} 
tung von Fragmentierungsannahmen befördert (Chander und Lê 2015; Hill 2012). Diese haben auch die praxisorientierte Internet-Governance-Szene beeinflusst (Drake et al. 2016; Meinrath 2013; kritisch: Mueller 2017).

Die in der Fragmentierungsdebatte aufscheinende harte Alternative zwischen der postnationalen und der nationalen Konstellation erscheint allerdings sowohl empirisch unzureichend als auch theoretisch defizitär. Saskia Sassen hat diese Erkenntnis bereits in ihrem „Paradox des Nationalen“ sehr präzise auch für die Digitalisierung formuliert: „Ein weitverbreitetes und - nach meiner Sicht - zutiefst fragwürdiges Verständnis stellt die digitalen Technologien als etwas dar, das das Territorium eliminiert und außerhalb nationaler Zuständigkeitsbereiche funktioniert, das von staatlicher Autorität unabhängig ist und sich daher dieser Autorität gegenüber potenziell subversiv verhält" (Sassen 2008, S. 519). Sassen hält der aus ihrer Sicht „technizistischen Lektüre“ der digitalen Revolution die vielfältigen Verflechtungen des Digitalen mit sozialen und kulturellen Gegebenheiten und Bindungen entgegen (Sassen 2008, S. 548).

Auch die jüngere politikwissenschaftliche Forschung hat sich der aufgezeigten Spannung zwischen Transnationalisierung und Fragmentierung differenzierter genähert, vornehmlich über das Konzept der Souveränität (mit Bezug zu den drei unten behandelten Beispielfeldern etwa: Drezner 2001; Shackelford et al. 2016; Hathaway 2014; Pistor 2020). Die in digitalpolitischen Diskursen zunehmend erkennbaren, diffusen Souveränitätskonzepte sind in einer Reihe von Arbeiten theoretisch unterfüttert und genealogisch umfassend untersucht worden (Pohle und Thiel 2019, 2020; Thiel 2019; Ritzi und Zierold 2019). Im Rahmen von Diskursanalysen konnten etwa für den deutschen Fall entsprechende Deutungsmuster rekonstruiert werden. In ähnlicher Weise haben andere Autor*innen auf das ebenfalls abstrakte Konzept der Territorialität gesetzt, um die politische Strukturierung von digitalpolitischen Diskursen und Praktiken (Möllers 2021; Lambach 2020) zu untersuchen.

Die genannten Arbeiten gehen in ihrem theoretischen und insbesondere empirischen Anspruch insofern über den hier verfolgten Ansatz hinaus, als die Leitkonzepte auch losgelöst vom Nationalstaaat funktionieren, sich etwa auf Vorstellungen individueller Autonomie im Umgang mit digitalen Medien und die „Territorialisierungsprojekte“ oder „Quasi-Souveränität“ anderer Akteure (wie etwa Plattformen) im digitalen Raum übertragen lassen (,quasi-sovereign“, Pohle und Thiel 2020, S. 7; „corporate territories“, Lambach 2020, S. 500). Die größere Reichweite ist insofern programmatisch, als das Verständnis der digitalen Konstellation jenseits hergebrachter Schablonen zur zeitgemäßen Aufgabe erklärt wird (Lambach 2020, S. 485). Dieser Beitrag folgt den terminologischen Extensionen nicht. Während er empirisch gut an zentrale der dort präsentierten diskurs- oder dispositivanalytischen Befunde anschließen kann, fokussiert er bewusst auf den auch für die offeneren Konzepte von Souveränität und Territorialität meist vorrangigen Bezug auf den nationalstaatlichen Rahmen (s. hierzu auch den Beitrag von Neyer in diesem Sonderheft). Denn gerade dieser fortwirkenden strukturellen Bindung beider Konzepte an den Nationalstaat soll nachgegangen, sie soll sichtbar gemacht und zum wesentlichen Erklärungsfaktor ausgebaut werden. Damit verspricht die Konzentration auf diesen vorrangigen Bezug an erster Stelle einen analytischen Gewinn gegenüber den breiteren und notwendigerweise diffuseren Souveränitäts- oder Territorialitätskonzepten 
an anderer Stelle. Zudem kann es als Vorteil des hier verfolgten Ansatzes betrachtet werden, dass er es nicht bei der gerade für die Diskursstudien typischen Rekonstruktion empirischer Muster belassen muss, sondern ein auf wissenssoziologischen (Strukturierung) oder politiktheoretischen (Legitimation) Erkenntnissen basierendes Erklärungsangebot für die beobachteten Phänomene unterbreitet.

\section{Nationalismusforschung und ihr vielschichtiges Theorieangebot für die digitale Konstellation}

Nationalismus ist ein vielgestaltiges Konzept. Es steht nicht allein für eine in sich bereits komplexe politische Idee (Kedourie 1971, S. 7), sondern bildet zugleich einen Sammelbegriff für weltanschaulich zusammenhängende, in ihrer ideologischen Ausgestaltung aber erstaunlich flexible, politische Bewegungen (Kohn 1955, S. 4). Diese konnten in den vergangenen zweieinhalb Jahrhunderten ein gesellschaftliches Organisationsmodell sowie eine politische Weltordnung etablieren, die auch für das 21. Jahrhundert noch prägend sind. Nationalismen stehen aufgrund der in ihrer historischen Entwicklung offenbar gewordenen Exklusionstendenzen und der Konfliktanfälligkeit des verschiedentlich aufgeladenen doktrinären Modells für verheerende Menschheitsverbrechen und Kriege (Connor 1993). Heute ist es also aus guten Gründen schlicht unmöglich, von Nationalismus zu sprechen, ohne negative Konnotationen hervorzurufen. Will man Nationalismustheorien auf aktuelle politische Entwicklungen anwenden und damit über die kritische Betrachtung neonationalistischer Bewegungen hinausgehen, muss allerdings von historischen und aktuellen nationalistischen Bewegungen abstrahiert werden. Was nach dieser Reduktion bleibt, ist immer noch komplex. Forscher*innen unterschiedlicher disziplinärer Provenienz haben das terminologische Repertoire über die Jahrzehnte der Beschäftigung beständig erweitert und ihm eine Vielzahl von ,Bindestrichnationalismen ' hinzugefügt (ausführliche Typologien bei Smith 2014; Werron 2018).

Im folgenden beziehe ich mich auf zwei übergeordnete Dimensionen, die ich als strukturell und doktrinär bezeichne. ${ }^{2}$ Strukturelle Nationalismen führe ich als Oberbegriff für solche Nationalismen ein, die uns gleichsam unterlaufen, die unserer Sprache, unserem Wissen und Handeln (unseren Diskursen und Dispositiven) eingeschrieben sind, die damit immer schon Wirkung entfalten, ohne dass wir uns ihrer in den meisten Fällen bewusst sind oder sie hinterfragen (Schünemann 2014, S. 512-518). Strukturelle Nationalismen sind zugleich Teil und grundlegende Konfigurationen gesellschaftlicher Wissensordnungen. Sie manifestieren sich in Diskursen

\footnotetext{
2 Zur Unterscheidung der in der Literatur verschiedentlich attribuierten Nationalismen ließe sich noch eine dritte Dimension der sog. emotionalen Nationalismen hinzufügen. Gemeint sind Nationalismen, die uns überkommen, die wir spüren. Hierzu gehören in erster Linie die im etwas undurchsichtigen Begriff des Patriotismus gefassten Gemütslagen. Gefühle dieser Art mögen uns bei internationalen Sportereignissen oder anderen Leistungswettbewerben (,Leistungnationalismus“ nach Werron 2018 auch im Bereich der Wirtschaft oder aktuell: Gesundheitspolitik), bei Berichten von nationalem Leid oder über historische Ereignisse, begegnen. Emotionale Nationalismen sind theoretisch am klarsten in der Sozialpsychologie zu verorten und empirisch unabweisbar (Billig 1995). Für die digitale Konstellation haben sie bislang augenschainlich nur geringe (womöglich katalytische) Bedeutung.
} 
und Praktiken. Auf sprachlicher Ebene treten sie als deiktische Marker und Bezugnahmen zum kollektiven Selbst und anderen in Erscheinung: „wir“, „uns“, ,,ihr“, „euch“, „hier“, „,dort“ usw. Sie zeigen sich mehr oder weniger prominent in nationaler Kollektivsymbolik wie Flaggen, Wappen, Allegorien oder Länderkürzeln. Sie sind eingeschrieben in gesellschaftliche Institutionen und institutionalisierte Praktiken. Schließlich treten sie besonders deutlich in eingeübten Narrativen hervor, durch die „Nationalformen des Wissens“ (Schünemann und Keller 2016, S. 61) reproduziert und prozessiert werden. Zum strukturellen Typ zähle ich dementsprechend deiktische, symbolische, institutionelle und narrative Nationalismen. In theoretischer Hinsicht verweisen die strukturellen Varianten auf ein wissenssoziologisches Feld (Berger und Luckmann 1969; Keller 2008). Offensichtliche Anknüpfungspunkte zeigen sich hier in konstruktivistischen Ansätzen der Nationalismustheorie (Anderson 2006; Hobsbawm 2005). Verwandte Konzepte sind auch unter Bezeichnungen wie „everyday“ (Fox und Miller-Idriss 2008) oder „banal nationalism“ (Billig 1995) behandelt worden.

Entgegen einer cyberutopisch oder postnational begründeten Überwindungsthese mit Blick auf den Nationalstaat, wie sie oben als Ausgangspunkt dieses Beitrags gedient hat, erlauben konstruktivistische Nationalismustheorien eine Erklärung für das Beharrungsvermögen nationalstaatlicher Ordnung unter veränderten Bedingungen in der zweiten Hälfte des 20. Jahrhunderts und zu Beginn des 21. Jahrhunderts. Die für diesen Beitrag zentrale Beharrungsthese kann auf die digitale Konstellation übertragen werden. Demnach stellen strukturelle Nationalismen einen wesentlichen Erklärungsfaktor für die nationalstaatliche Strukturierung digitalpolitischer Regulierung und für aktuelle Ordnungskonflikte dar (s. auch Möllers 2021, S. 121).

Strukturelle Nationalismen stehen also für Beharrlichkeit. Ihnen fehlen die eigenen Antriebskräfte. Sie sind nicht eigendynamisch, nicht progressiv (also gerade keine ,,aktive Kraft“ im Sinne Kohns 1955, S. 17), sondern allenfalls formerhaltend. Für neuen Schub und Legitimation in Ordnungskonflikten sind sie auf andere Varianten angewiesen. Hier kommen in erster Linie doktrinäre Formen ins Spiel. Doktrinäre Nationalismen („Nationalismus als Doktrin“, Kedourie 1971) sind solche Nationalismen, die uns überzeugen, für die eingetreten, gestritten, womöglich gar gekämpft wird. Auch in dieser Klasse lassen sich verschiedene Varianten unterscheiden. An erster Stelle und am theoretischen Ursprung stehen autonomistische Nationalismen. Vorstellungen von Autonomie und Selbstbestimmung bilden den doktrinären Kern von Nationalismus überhaupt. Nationalismus ist, wie es bei Kedourie (1971, S. 30) heißt, ,im wesentlichen eine Doktrin der nationalen Selbstbestimmung“. Gerade in der Betonung von Autonomie (nach innen wie nach außen) wird die Qualität von Nationalismustheorie als Theorieangebot der Moderne ersichtlich (Gellner 1999). Indem sie die naturrechtlich gebotene Autonomie des Individuums auf einen nur dadurch begründeten abstrakten politischen Verband übertragen hat, hat die Idee des Nationalismus den in der Modernisierungskrise erforderlichen politischen und legitimatorischen Paradigmenwechsel angetrieben und untermauert. Der Gedanke gesellschaftlicher Autonomie macht den gemeinsamen geistesgeschichtlichen Ursprung mit modernen demokratisch-egalitären Vorstellungen sichtbar. Andere doktrinäre Varianten (religiös, rassistisch, faschistisch) haben sich gerade zu dieser ursprünglichen Verwandtschaft immer wieder in verheerende Opposition gestellt. Dabei muss 
freilich klargestellt werden, dass die Unterscheidung zwischen strukturellen und doktrinären Varianten eine idealtypische ist. Schnittmengen ergeben sich in der Realität gerade an den Übergängen zwischen beiden Typen. So gehen doktrinäre Nationalismen (etwa religiöse oder faschistische) mit einer markanten Kollektivsymbolik und spezifischen Narrationen einher, die sich tief in gesellschaftliche Wissensbestände und kulturelle Codes einschreiben können.

Während doktrinäre Nationalismen in historischer Betrachtung überhaupt konstitutiv für alle strukturellen Nationalismen gewesen sind, kann man heute - zumindest für stabile Nationalstaaten - überwiegend von einem umgekehrten Verhältnis ausgehen. Demnach bieten strukturelle Nationalismen die Anknüpfungspunkte, an denen doktrinäre Varianten andocken und sich ausbilden (oder reanimieren) können (Werron 2018, S. 52). Ich bezeichne diese zweite zentrale Annahme dieses Beitrags in Anlehnung an Werron als Anknüpfungsthese. Die Kombination aus strukturellen und doktrinären Varianten hat - so möchte ich im Folgenden argumentieren Erklärungskraft für digitalpolitische Entwicklungen unserer Zeit und damit für die Ausgestaltung der digitalen Konstellation (eine Seite des von Berg et al. aufgezeigten theoretischen Erkenntnisinteresses 2020, S. 183).

\section{Zur Bedeutung struktureller und doktrinärer Varianten für ausgewählte digitalpolitische Felder}

Im Folgenden möchte ich die oben skizzierten nationalismustheoretischen Annahmen an drei digitalpolitischen Beispielen überprüfen: nämlich der Regulierung kritischer Internetressourcen am Beispiel des Domain-Name-Systems (DNS), an der Ausbildung von Cybersicherheitspolitiken in Europa und den USA sowie schließlich der medienpolitischen Regulierung von Online-Inhalten im Hinblick auf Desinformation. Mit der Fallauswahl möchte ich sowohl verschiedene Dimensionen der politischen Institutionalisierung von Internet und Digitalisierung abdecken: nämlich eine Konstitutions-, eine Konflikt- und eine Kontrolldimension, die von nationalstaatlichen Ordnungsvorstellungen in unterschiedlicher Weise berührt sind. Zudem ermöglicht die Fallauswahl auch einen Blick auf unterschiedliche Epochen der Internetentwicklung, ausgehend von den 1980er- und 1990er-Jahren im Hinblick auf das DNS, auf die darauffolgenden zwei Dekaden im Hinblick auf die Cybersicherheitspolitik und auf die aktuellen Entwicklungen mit dem Beispiel Desinformationsbekämpfung.

\subsection{Unser Teil vom Cyberspace - die partiell nationale Strukturierung des Domain-Name-Systems}

Eine der eindrücklichsten und zugleich unverdächtigsten strukturell-nationalistischen Manifestationen spiegelt sich bis heute in unserer täglichen Internetnutzung, konkret in der Adresszeile jedes Internetbrowsers in Form der Top-Level-Domains (TLDs), wider. Das DNS, wie wir es bis heute kennen und gebrauchen, bildete sich mit der allmählichen Reifung des Internets zu einem Massenkommunikationsmittel heraus. Die Verwaltung der kritischen Ressourcen des Internets lag in den 1980er- 
Jahren noch weitgehend in den Händen eines Kreises von Entwickler*innen, der die notwendigen Standards setzte und die auf dieser Grundlage vergebenen Adressen und Namen autoritativ verwaltete. Das dynamische Wachstum des Internets drohte rasch die organisch entstandenen Strukturen organisatorisch, aber auch legitimatorisch zu überfordern. Vor diesem Hintergrund entwarfen die für dieses Dossier zuständigen Mitglieder des Entwickler*innenkreises das DNS in seiner grundlegenden Struktur (Postel und Reynolds 1984). Demnach wurde auf der obersten Hierarchieebene eine Kombination aus zwei verschieden geordneten Zeichensystemen etabliert, nämlich einem System sog. generischer TLDs, die gesellschaftliche Teilsysteme und Organisationstypen repräsentieren (zunächst fünf: „COMmerce“, „EDUcation“, „GOVernment“, „MILitary“, „ORGanization“) und zumindest ursprünglich keinerlei territorialstaatlicher Bindung unterlagen, ${ }^{3}$ sowie dem an der nationalstaatlichen Weltordnung und international anerkannten Bezeichnungsstandards orientierten Schema: den sog. country-code TLDs (ccTLDs). Die LänderCodes lagen in Form eines internationalen Standards vor, so dass die Entwickler*innengemeinde mit dem Rückgriff auf die entsprechende Liste der International Standards Organisation (ISO-Liste 3166) auch die Verantwortung für womöglich kontroverse Zuordnungen vermied.

Mit dem Einschreiben des nationalstaatlichen Zeichensystems in die Konstitution des Internets als soziotechnischem Raum - denn für das Funktionieren eines Rechnernetzwerks braucht es keine sinntragende Symbolik - traf der Entwickler*innenkreis eine semantisch und politisch folgenschwere Entscheidung. Sie lässt sich strukturell-nationalistisch erklären. Selbst für die kosmopolitisch orientierte Arbeitsgruppe lag der Rückgriff auf das nationalstaatliche Ordnungsschema gerade in dem Zeitraum, in dem die Entwicklung des Internets die technische und organisatorische Grenzüberschreitung über den US-Kontext hinaus verlangte, nahe. Aus dem tief eingeschriebenen deiktischen Nationalismus von Hier- und Dort-, Innen- und Außen-Bezügen wurde eine symbolische Ordnung.

Es ist gewiss zu diskutieren, welche Bedeutung das DNS in seiner grundlegenden Architektur für die heutigen Nutzungsweisen des Internets und die über das Netz hinausgehenden Digitalisierungsprozesse noch hat, und doch taugt das Beispiel als ein erstaunlicher Beleg für die oben formulierte Beharrungsthese. Darüber hinaus bieten die sich aus der Strukturentscheidung ergebenden Entwicklungen Illustrationen für die Anschlussfähigkeit doktrinärer Nationalismen im Sinne der Anknüpfungsthese.

Einen ersten Moment dieser Art bildet die politische Institutionalisierung der Verwaltung der kritischen Internetressourcen einschließlich des DNS Ende der 1990erJahre. Sie mündete in ein neuartiges und betont staatsfernes, globales GovernanceArrangement im Sinne des Multi-Stakeholder-Modells unter der Ägide der ICANN (International Corporation for Assigned Names and Numbers). Dabei wurde die nationalstaatliche Kontestation des Modells vor allem über die Bildung eines Governmental Advisory Committee (GAC) innerhalb der Institution kanalisiert, dem allerdings nur eine marginale und beratende Funktion in Bezug auf die wesentlichen

\footnotetext{
3 Dies änderte sich in den 1990er-Jahren für die TLDs .EDU, .GOV und .MIL, die die USA für sich reklamierten. Schon darin scheint ein autonomistischer Nationalismus auf, wie er im Sinne der Anknüpfungsthese zu erwarten ist.
} 
Entscheidungskompetenzen zugesprochen wurde. Mit den ccTLDs stand eine symbolische Ressource bereit, die anschlussfähig war an die autonomistischen Vorstellungen vieler nationalstaatlicher Mitglieder des GAC. Mit den sog. GAC-Prinzipien (ICANN/GAC 2000) formulierten die Mitgliedstaaten ihren souveränen Anspruch auf ihre ccTLD und brachten zugleich die symbolische Aufladung zum Ausdruck: „ccTLDs have acquired an increasing part in the domain names market and are seen by many as part of the Internet identities of their country or geopolitical territory“" (ebd.).

Auf nationaler Ebene setzte sich der angesprochene Mechanismus in international varianter Form mit Blick auf die Verwaltungsarrangements für die ccTLDs fort. In einer von Geist (2004) für die ITU (International Telecommunication Union) durchgeführten vergleichenden Studie zeigt sich, dass die Wahrnehmung der ccTLDs als genuin hoheitliche symbolische Ressource der Nationalstaaten unter den Regierungen weit verbreitet war. In einer jüngeren Analyse eines Rechtsstreits über den Entzug der iranischen ccTLD illustrieren Mueller und Badiei (2017) die weitgehende Orientierung rechtswissenschaftlicher und rechtlicher Bewertungen zugunsten staatlicher Ansprüche. Wie verbreitet die doktrinär-nationalistischen Aufladungen der ccTLDs sind, zeigt sich auch in der wissenschaftlichen Diskussion, etwa mit Blick auf den Handel mit attraktiven ccTLDs (Drezner 2001; Hrynyshyn 2008).

Schließlich sei auf die verschiedenen und prominent behandelten identitätspolitischen Projekte hingewiesen, die auch auf dem Feld des DNS ausgetragen wurden und werden. So sind TLDs in der Vergangenheit zum symbolischen Ausdruck nationalen Unabhängigkeitsstrebens geworden wie im Falle Kosovos (Randazza 2016) oder auch der spanischen Provinz Katalonien (Gerrand 2006). Selbst die Anstrengungen der EU für den Erhalt einer quasi-ccTLD ,.EU` hat - wenngleich nicht nationalistisch - in der identitätspolitischen Aufladung der Zeichenkette Ähnlichkeit mit den anderen Beispielen.

Insgesamt lässt sich für dieses erste Beispiel der ccTLDs festhalten, dass die Beharrungsthese eine strukturell-nationalistische Erklärung für die ansonsten schwer zu motivierende Wahl eines Systems sinntragender Länderkürzel für die oberste Domain-Ebene der Internet-Adressierung bietet. Darüber hinaus sind die darauf folgenden internationalen Auseinandersetzungen um die Verwaltung der ccTLDs, die politische Institutionalisierung der Domain-Verwaltung auf nationaler Ebene sowie die Bedeutung von ccTLDs im Rahmen nationalistischer Identitätspolitiken Anzeichen für die Gültigkeit der Anknüpfungsthese.

\subsection{Es geht um unsere Sicherheit - Bedrohungswahrnehmung und erzähltes Konfliktgeschehen im Cyberraum}

Cybersicherheitspolitische Debatten sind in hohem Maße von Unsicherheit geprägt. Gegenüber anderen Sicherheitsbedrohungen ergibt sich die gesteigerte Unsicherheit mindestens aus der Virtualität der Angriffstechniken und den mangelnden kinetischen Effekten, aus der weltweiten Vernetzung und dem globalen Datenverkehr sowie der mit beidem verbundenen chronischen Attributionsproblematik, wobei die besseren Möglichkeiten verdeckter Operationen und der Verschleierung einem Angreifer ermöglichen, die Urheberschaft für eine Tat zu leugnen (plausible 
deniability). Die Bedrohungswahrnehmung, einschließlich der Einschätzung gesellschaftlicher Verwundbarkeiten, geeigneter Defensivmaßnahmen und die Attribution gegenüber Offensivakteuren speisen sich mangels gesicherter Erkenntnisse aus gesellschaftlichen Wissensbeständen. In diese sind strukturelle Nationalismen tief eingeschrieben. Neben einfachen deiktischen Bezügen von hier und dort, innen und außen sind es zunächst institutionelle Nationalismen, die sicherheitspolitische Diskurse und Dispositive allgemein prägen und sich auch in der Cybersicherheitspolitik niederschlagen. Dies lässt sich etwa an der Definition kritischer Infrastrukturen, verstanden als die notwendigen Infrastrukturen für das Funktionieren moderner, d. h. in aller Regel nationaler, Gesellschaften (Dunn Cavelty 2013), ablesen, die einen Grundstein nationaler Cybersicherheitsstrategien bildet.

Seit dem Auswachsen des Internets zu einer gesellschaftsdurchdringenden Kommunikationsinfrastruktur wurde parallel zu den alltäglichen Routinen der IT-Sicherheit eine politische Debatte über die höchsten Eskalationsdynamiken geführt, wobei die realen Vorfälle bislang weitgehend hinter den katastrophenförmigen Szenarien zurückgeblieben sind (Rid 2018). Dieser Strang der Cybersicherheitsdebatte basiert gewissermaßen auf der ,erzählten Katastrophe“ (Dunn Cavelty 2013) von kinetischen Folgen und verheerenden Störungen kritischer Infrastrukturen. Politische Entscheidungsträger*innen haben auf die veränderte Bedrohungsperzeption reagiert, indem sie für die Anbieter kritischer Infrastrukturen besondere Schutzziele definiert haben und die geforderte Absicherung durch staatliche Zertifizierung und Behördenaufsicht kontrolliert wird (Schünemann 2020, S. 205-206). Trotz regionaler Harmonisierung etwa im EU-Rahmen (NIS-Richtlinie: EU 2016/1148), bleibt die eigentliche sicherheitspolitische Funktionsdefinition national ausgerichtet. Der sicherheitspolitische Fokus auf die kritischen Infrastrukturen verpflichtet bestimmte Industriezweige auf den Schutz nationaler Sicherheitsinteressen und stabilisiert damit strukturelle Schließungen inter-nationaler Sicherheitsarchitektur im Sinne der Beharrungsthese (dazu aus Perspektive der Sekuritisierungstheorie Nissenbaum 2005).

Auch die Debatten um den Schutz kritischer Infrastrukturen bergen Anknüpfungsmöglichkeiten für doktrinäre Nationalismen. Besonders sichtbar wird dieser Zusammenhang, wo die Netzwerkinfrastruktur selbst in den Fokus rückt. Gerade in Europa ist die wachsende Abhängigkeit von ausländischen Anbietern von Informations- und Kommunikationstechnologien für die Bereitstellung grundlegender digitaler Kommunikationsinfrastrukturen im vergangenen Jahrzehnt wiederholt zum Thema gemacht worden. Nach den Enthüllungen geheimdienstlicher Überwachungspraktiken durch den Whistleblower Edward Snowden wurde insbesondere die Abhängigkeit gegenüber den Vereinigten Staaten problematisiert und wurde mit dem sog. Schengen-Routing der Aufbau zumindest einer auf europäischer Ebene besser kontrollierbaren eigenen Netzwerkinfrastruktur ins Spiel gebracht. Die aktuelle deutsch-französische Initiative zum Aufbau einer europäischen Cloud-Infrastruktur unter dem Titel Gaia-X (BMWi 2021; Pohle und Thiel 2020: 10) knüpft an die autonomieorientierten Deutungsmuster an und verbindet die Vision infrastruktureller Unabhängigkeit mit wirtschaftspolitischen Entwicklungszielen. Wenngleich die genannten Initiativen gerade nicht auf den nationalstaatlichen Rahmen beschränkt sind und damit Vorstellungen von Souveränität und Territorialität jenseits des Nationalstaats transportieren, entsprechen sie doch den bekannten doktrinären Mustern, 
die sich außerhalb des europäischen Rahmens auch auf nationalstaatlicher Ebene aktualisieren.

Dies zeigen die jüngere Debatte und die konkreten Regulierungsschritte zur Verbannung chinesischer Unternehmen wie Huawei aus dem US-amerikanischen Telekommunikationsmarkt im Rahmen der Clean-Network-Initiative unter Ex-Präsident Trump. Sie hat erhebliche Folgewirkungen für europäische Partnerstaaten - der Initiative haben sich über 50 Staaten, einschließlich der NATO-Staaten, angeschlossen. In ihren teils schwierigen Entscheidungsfindungen zum Ausschluss von Huawei beim Ausbau der Mobilfunknetze zum 5G-Standard folgen sie einer Bedrohungswahrnehmung, die auf den eingeübten Narrativen einer internationalen Konfliktkonstellation beruht und eine territorialstaatliche Sicherheitsarchitektur und Verantwortungszuschreibung verfestigt (Möllers 2021, S. 122). In gewissem Kontrast zur nationalen Schließung steht zwar der multilaterale Charakter der Initiative, deren primäres Ziel - positiv gewendet - im Erhalt einer transnational vertrauenswürdigen Netzwerkinfrastruktur unter demokratischer Kontrolle gesehen werden kann. Doch auch diese Perspektivverschiebung in Richtung eines geopolitischen Systemkonflikts um die Governance der Digitalisierung hält Ansatzpunkte für strukturelle und doktrinäre Nationalismen bereit.

Zusammengefasst prägen institutionelle Nationalismen und narrative Nationalismen die Cybersicherheitspolitik sowohl auf der domestischen als auch der internationalen Ebene. Sie tragen zur Stabilisierung nationalstaatlicher Ordnung im Sinne der Beharrungsthese bei. Mit den jüngeren Debatten um technologische Souveränität knüpfen autonomistische Vorstellungen erkennbar an strukturell-nationalistische Bedrohungswahrnehmungen an und können als Beispiele für die Anknüpfungsthese dienen.

\subsection{Bitte nicht stören! - nationale Autonomie in der Desinformationsordnung}

Die seit 2016 mit großer Intensität geführte gesellschaftliche Debatte über digital verbreitete Falschnachrichten und die daraus resultierenden Gefährdungen für die Demokratie in einer neuen ,Desinformationsordnung“ (Bennett und Livingston 2018) haben viele diskursive Verbindungslinien zu den oben skizzierten Strängen der Cybersicherheitsdebatte, sowohl im Hinblick auf die Bedrohungswahrnehmung als auch die Politikdefinition. Dies wird besonders deutlich in der Problematisierung manipulativer Störungen von nationalen Wahlkämpfen als Begleiterscheinung transnationaler Öffentlichkeiten (Pfetsch et al. 2019, S. 95). Irritierende Wahl- und Abstimmungsergebnisse der jüngeren Vergangenheit (insb. das Brexit-Referendum und die Wahl Donald Trumps zum US-Präsidenten) werden zumindest teilweise oder mittelbar auf die manipulativen Eingriffe zumeist ausländischer Akteure in nationale Öffentlichkeiten zurückgeführt (Jamieson 2018). Geheimdienstberichte haben die entsprechenden Verdachtsmomente verschiedentlich gestützt. Die Informationsoperationen können zwar in einen weiteren historischen Kontext geheimdienstlicher Praktiken verschiedener Staaten gestellt werden (Buchanan 2020, S. $211 \mathrm{ff}$.; Rid 2020; Thornton und Miron 2019, S. 258). Der strategische Nutzen sowie die Effekte von Desinformationskampagnen etwa auf die Wahlbevölkerung in komplexen Medien- und Informationssystemen sind überdies weiterhin unklar (Lanoszka 2019, 
S. 233). Und doch sind sog. ,,interferences“, also die manipulativen Eingriffe ausländischer Staaten oder sog. Proxies in nationale Öffentlichkeiten, zu einem zentralen Baustein demokratischer Bedrohungswahrnehmung in der digitalen Konstellation geworden. Die politische, aber auch die wissenschaftliche Diskussion thematisieren dabei neuerdings gerade die asymmetrische Verwundbarkeit von Demokratien in einem globalen Umfeld mit freien Informationsflüssen und entwerfen medienregulatorische oder sicherheitspolitische Maßnahmen für Schutz und Gegenwehr (Farrell und Schneier 2018; Goldsmith und Russell 2018). Die Phänomenstruktur der Bedrohung verläuft klar entlang den Demarkationslinien einer national strukturierten Welt- und Konfliktordnung. Schon der englischsprachige Ausdruck der ,interferences“ macht diese strukturell-nationalistische Orientierung im Sinne der Beharrungsthese sichtbar.

Neben den institutionellen Bezügen auf den Nationalstaat in den Sicherheitsund Kontrollarchitekturen fallen vor allem die narrativen Nationalismen als grundlegende sinnstiftende und plausibilisierende Strukturen für irritierende politische Ereignisse auf. Nicht zuletzt die kontroversen Diskussionen über die Bedeutung ausländischer Desinformationskampagnen für die Ausgänge des Brexit-Referendums und der Wahl Trumps 2016 können hier als Beispiele dienen. Die Annahme einer staatlich orchestrierten und kontrollierten Kampagne gegen die nationale Sicherheit fügt sich nicht allein in die eingeübten Narrative internationaler Politik, sondern kompensiert gleichsam für die durch Wahl- und Abstimmungsergebnisse verletzten Rationalitätserwartungen. Thomas Rid legt in seinem Buch „Active Measures“ (Rid 2020) vor dem Hintergrund jahrzehntelang praktizierter geheimdienstlicher Informationsoperationen für die russischen Aktivitäten dar, inwiefern die unterstellte Verquickung staatlicher Behörden zwar vielfach zutrifft, aber die Wahrnehmung perfekt orchestrierter, gezielter Kampagnen dennoch weit über die Realität hinausgeht. Narrative Nationalismen befördern jedoch ein auf gewohntes Rollenverhalten zugespitztes Verständnis der Bedrohungslage vor der Folie bekannter Konfliktkonstellationen. Möllers hat auf anderem Weg herausgearbeitet, inwiefern die in diese Narrative eingeschriebenen Rollenzuschreibungen etwa die Cybersicherheitspolitik Deutschlands prägen:
Although there are some overlaps, „Chinese hackers“ are mostly associated with economic espionage; ,Russian hackers“ are associated with political es- pionage, manipulation, and disinformation campaigns; and „Islamic terrorists“ are associated with sabotage of vital systems. In other words, China is predomi- nantly framed as a threat to the national economy, Russia is framed as a threat to German political institutions, and Islamic terrorists are framed as threats to vital systems and life itself. (Möllers 2021, S. 122; s. auch S. 123)

Narrative Nationalismen halten nicht nur anpassungsfähige Schablonen für die schlüssige - nationale - Attribution diffuser Informationsoperationen nach Cui-bono-Logik bereit, sie schaffen zugleich eine legitimatorische Grundlage für staatliche Eingriffe in die Informationsfreiheit. Frankreichs gesetzgeberische Antwort auf die Desinformationskampagnen im Präsidentschaftswahlkampf 2017 etwa, das sog. loi infox, ein im europäischen Kontext neuartiges Gesetz gegen Falschnachrichten aus dem Jahr 2018, kann hier als Beispiel dienen. Das Gesetz sieht eine Ver- 
pflichtung großer sozialer Netzwerke vor, die Verbreitung von Falschnachrichten in Wahlkampfzeiten (drei Monate bis Wahltermin) verstärkt zu bekämpfen, einschließlich richterlicher Löschanordnungen. Präsident Macron hat in seiner Vorstellung des Gesetzesvorhabens an der Verantwortungszuschreibung im Sinne internationaler Konfliktlogik keinen Zweifel gelassen und die Angriffe in den Kontext eines übergeordneten politischen Systemkonflikts gestellt:

Die Zunahme von Falschnachrichten dieser Tage ist eng verbunden mit der illiberalen Faszination, die ich hier erwähne, weil sie oftmals von den gleichen Kräften finanziert und genutzt wird, die sich in gewisser Weise über die Schwächen der Demokratie lustig machen [...]. ${ }^{4}$ (Übersetzung aus dem Franz. durch den Verf., Macron 2018)

Macron und andere Regierungspolitiker*innen reformulierten in ihren Rechtfertigungen der ungewöhnlichen Eingriffe in die Informationsfreiheit nicht allein strukturell-nationalistische Muster. Mit dem Schutz demokratischer Wahlen und Wahlkämpfe vor ausländischen Einflüssen wurde zudem die demokratische Souveränität thematisiert. In dem aus der Bedrohungswahrnehmung resultierenden Ruf nach zumindest temporär hochgezogenen Schutzwällen für nationale Kommunikationsräume in Wahlkampfzeiten - auf dass sich die demokratische Willensbildung unverfälscht vollziehen könne - offenbart sich damit nicht allein eine besonders große Spannung zur transnationalen Konnektivität als konstitutiver Konstante der digitalen Konstellation, sondern auch ein besonders deutlicher Anwendungsfall für die Anknüpfungsthese. In den autonomistischen Erwartungen, zumindest in Wahlkampfzeiten einen Kommunikationsraum gegen äußere Einflüsse partiell abzuschotten, gelangt die nationalistische Idee in zweierlei Hinsicht zu ihrem doktrinären Kern: nämlich sowohl im Hinblick auf die Selbstbestimmung der Bevölkerung als auch im Hinblick auf die Legitimation von Herrschaft und ihrer Kontrolltechniken, hier in Form der Regulierung von Internetinhalten. In der Erwartung eines von ausländischen Einmischungen möglichst unbeeinträchtigten Wahlakts wird der Anspruch auf nationale Autonomie besonders deutlich. Das von Ernest Gellner (1999) auf den Punkt gebrachte „Hauptanliegen“ des Nationalismus: „Einfach ausgedrückt heißt das: Kein Ausländer soll über uns herrschen!“, kommt in den aktuellen Debatten um Desinformationskampagnen und geeignete Gegenwehr auch in liberalen Demokratien zumindest mit Blick auf die Hochphasen politischer Willensbildung zum Vorschein und trifft augenscheinlich auf Akzeptanz.

Zuletzt fallen also auch die medienregulatorischen und sicherheitspolitischen Debatten über den Umgang mit Desinformation durch strukturell-nationalistische Deutungsmuster und Narrative auf. Das daraus abgeleitete Schutzbedürfnis demokratischer Öffentlichkeiten und die Legitimation regulatorischer Eingriffe in die Informationsfreiheit lassen autonomistische Vorstellungen erkennen. Damit ergeben die aktuellen Reaktionen auf die sog. Desinformationsordnung auch in liberalen Demokratien einen besonders virulenten Anwendungsfall für die Anknüpfungsthese.

\footnotetext{
4 „Cette montée des fausses nouvelles est aujourd'hui totalement jumelle de cette fascination illibérale que j'évoquais puisqu'elle est bien souvent financée par les mêmes, elle est bien souvent utilisée par des puissances qui s'amusent en quelque sorte des faiblesses de la démocratie [...]“.
} 


\section{Aufwärtskompatibel? Ein Fazit}

In den vorangegangenen Abschnitten wurde der Versuch unternommen, wesentliche Erkenntnisse aus der Nationalismusforschung auf die digitale Konstellation zu übertragen, um einen theoretischen Beitrag zum zunehmend deutlichen Spannungsverhältnis zwischen transnationalen Potenzialen und nationaler Strukturierung zu leisten. Mit der Kombination aus strukturell- und doktrinär-nationalistischen Zusammenhängen und Mechanismen konnten Erklärungsangebote für strukturelle Beharrlichkeit und politische Bedeutung des Nationalen für die Konfiguration der digitalen Konstellation unterbreitet und beispielhaft illustriert werden.

Im Ergebnis sollten transnationaler Einsatz und die Entwicklung digitaler Technologie nicht dazu verleiten, der digitalen Konstellation a priori eine transnationalisierende Entwicklungsrichtung zuzuschreiben. Umgekehrt ist auch das theoretische Angebot aus der Nationalismusforschung weder normativ noch deterministisch zu verstehen. Dass Nationalismen sich insofern als aufwärtskompatibel erweisen, als sie auch an die digitale Konstellation anschlussfähig sind, heißt nicht, dass jene notgedrungen einem nationalstaatlichen Ordnungsmodell folgt. So zeigt insbesondere das Beispiel der EU auf Ebene regionaler Governance, dass ehrgeizige digitalpolitische Ambitionen jenseits von Nationalstaatlichkeit erfolgreich verfolgt werden können. Mit der Europäischen Datenschutzgrundverordnung und künftig mit einer Kombination aus Digital Services Act und Digital Markets Act hat die EU potenziell regulatorische Innovationen vorgeschlagen, die über den nationalstaatlichen Rahmen hinausweisen, die globale Standards zu setzen, die führenden Plattformen zu disziplinieren suchen und insbesondere der Fragmentierung digitaler Kommunikationsräume durch einzelstaatliche Politiken zuvorkommen sollen.

Zudem ist die Betrachtung der digitalen Konstellation hier vornehmlich auf eine ihrer Kernkomponenten - die Internetentwicklung - reduziert worden. Die digitale Konstellation geht jedoch über die informationstechnologische Vernetzungsdimension der Digitalisierung hinaus und schließt insbesondere die Dimensionen der Datafizierung und Algorithmisierung sowie die mit ihnen einhergehenden soziotechnischen Veränderungen ein (Berg et al. 2020). Es wird zu untersuchen sein, inwieweit die dadurch erfassten Phänomene des Wandels nationalistische Denkmuster irritieren oder replizieren. Zumindest der Empirismus und die erfahrungsbasierte Modellierung einer an Big Data und algorithmischen Entscheidungen orientierten Epistemologie (O'Neil 2017, S. 15ff..) scheinen die Beharrlichkeitsbedingungen struktureller Nationalismen auf den ersten Blick nicht wesentlich herauszufordern. Inwieweit die in der Herausbildung befindlichen Regulierungsansätze für Künstliche Intelligenz derlei Prädispositionen durch universalistische und transnationale Leitlinien relativieren oder im Gegenteil durch zusätzliche doktrinär-nationalistische Programmatik gar verstärken, ist eine offene Frage.

Was bedeuten die in den Beispielen aufgezeigten schwächeren und stärkeren Lebenszeichen des Nationalen aber für die Vernetzungsdimension der digitalen Konstellation? Insbesondere die jüngeren Beispiele aus der Cybersicherheitspolitik sowie Medienpolitik im Bezug auf die sog. Desinformationsordnung sprechen für nationale Schließungstendenzen, die transnationale Konnektivität sowie staatsferne Governance infrage stellen und auch universelle Rechte von Nutzer*innen gefährden 
können. In dem Maße, in dem strukturell-nationalistische Denkweisen von doktrinär-nationalistischen Forderungen gedeckt und verstärkt werden, drohen sich diese Entwicklungen zu verschärfen. Dies bedeutet zwar nicht die Fragmentierung des Internets als technischer Infrastruktur, wohl aber eine Reorganisation im Sinne eines politischen „Alignment“, wie es Milton Mueller (2017) als realistisches Szenario beschreibt. Damit könnten die Beharrlichkeit struktureller Nationalismen und die Anknüpfungsmöglichkeiten doktrinärer Varianten, wie sie in den vorangegangenen Abschnitten illustriert worden sind, die derzeit virulente politische Institutionalisierung von Internet und Digitalisierung prägen und damit nachhaltige Effekte auf die Digitale Konstellation haben.

Im IT-Diskurs bezeichnet Aufwärtskompatibilität den Umstand, dass eine ältere Software- oder Hardware-Komponente auch in einer neuen Umgebung (oder Konstellation) noch funktioniert. Was aus Sicht von Anwender*innen gut klingt, kann aus Perspektive der Systementwicklung indes auch kritisch betrachtet werden. So impliziert das Konzept der Kompatibilität über Zeit immer auch eine Rückwärtsgewandtheit des Systems, die sein theoretisches Erneuerungspotential zugunsten praktischer Konventionen und Beharrlichkeitserwartungen der Anwender*innen einschränkt. Die Ambivalenz der Metapher kann auf das abschließende Fazit übertragen werden: Denn während sich das Theorieangebot der Nationalismusforschung für die digitale Konstellation als kompatibel und fruchtbar erweist, macht die Kompatibilität von Nationalismen ihre Überwindung im Rahmen der Digitalen Konstellation unwahrscheinlich.

Funding Open Access funding enabled and organized by Projekt DEAL.

Open Access Dieser Artikel wird unter der Creative Commons Namensnennung 4.0 International Lizenz veröffentlicht, welche die Nutzung, Vervielfältigung, Bearbeitung, Verbreitung und Wiedergabe in jeglichem Medium und Format erlaubt, sofern Sie den/die ursprünglichen Autor(en) und die Quelle ordnungsgemäß nennen, einen Link zur Creative Commons Lizenz beifügen und angeben, ob Änderungen vorgenommen wurden.

Die in diesem Artikel enthaltenen Bilder und sonstiges Drittmaterial unterliegen ebenfalls der genannten Creative Commons Lizenz, sofern sich aus der Abbildungslegende nichts anderes ergibt. Sofern das betreffende Material nicht unter der genannten Creative Commons Lizenz steht und die betreffende Handlung nicht nach gesetzlichen Vorschriften erlaubt ist, ist für die oben aufgeführten Weiterverwendungen des Materials die Einwilligung des jeweiligen Rechteinhabers einzuholen.

Weitere Details zur Lizenz entnehmen Sie bitte der Lizenzinformation auf http://creativecommons.org/ licenses/by/4.0/deed.de.

\section{Literatur}

Anderson, Benedict. 2006. Imagined communities: reflections on the origin and spread of nationalism. London: Verso.

Barlow, John Perry. 1996. A declaration of the independence of cyberspace. https://projects.eff.org/ barlow/Declaration-Final.html. Zugegriffen: 13. März 2015.

Benkler, Yochai. 2006. The wealth of networks. How social production transforms markets and freedom. New Haven: Yale Univ. Press.

Bennett, W. Lance, und Steven Livingston. 2018. The disinformation order: disruptive communication and the decline of democratic institutions. European Journal of Communication 33(2):122-139. 
Berg, Sebastian, Niklas Rakowski, und Thorsten Thiel. 2020. Die digitale Konstellation. Eine Positionsbestimmung. Zeitschrift für Politikwissenschaft 30(2):171-191.

Berger, Peter L., und Thomas Luckmann. 1969. Die gesellschaftliche Konstruktion der Wirklichkeit. Eine Theorie der Wissenssoziologie. Frankfurt/Main: Fischer.

Billig, Michael. 1995. Banal nationalism. London/Thousand Oaks: SAGE.

BMWi. 2021. GAIA-X: Eine vernetzte Datenstruktur für ein europäisches digitales Ökosystem. https:// www.bmwi.de/Redaktion/DE/Dossier/gaia-x.html. Zugegriffen: 27. Febr. 2021. Bundesministerium für Wirtschaft und Energie.

Buchanan, Ben. 2020. The hacker and the state: cyber attacks and the new normal of geopolitics. Cambridge: Harvard University Press.

Castells, Manuel. 2001. Der Aufstieg der Netzwerkgesellschaft. Opladen: Leske \& Budrich.

Chander, Anupam, und Uyên P. Lê. 2015. Data nationalism. Emory Law Journal 64(3):677-739.

Connor, Walker. 1993. Ethnonationalism: the quest for understanding. Princeton: Princeton University Press.

DeNardis, Laura. 2014. The global war for internet governance. New Haven/London: Yale University Press.

van Dijck, José, Thomas Poell, und Martijn de Waal. 2018. The platform society: public values in a connective world. New York: Oxford University Press.

Drake, William J., Vinton G. Cerf, und Wolfgang Kleinwächter. 2016. Internet fragmentation: an overview. http://www3.weforum.org/docs/WEF_FII_Internet_Fragmentation_An_Overview_2016.pdf. Zugegriffen: 27. Febr. 2021.

Drezner, Daniel W. 2001. Sovereignty for Sale. Foreign Policy 126:76-77.

Dunn Cavelty, Myriam. 2013. Der Cyber-Krieg, der (so) nicht kommt: Erzählte Katastrophen als (Nicht)Wissenspraxis. In Aufbruch ins Unversicherbare. Zum Katastrophendiskurs der Gegenwart, Hrsg. Leon Hempel, Marie Bartels, 209-233. Bielefeld: transcript.

Farrell, Henry John, und Bruce Schneier. 2018. Common-knowledge attacks on democracy. SSRN Electronic Journal. https://doi.org/10.2139/ssrn.3273111.

Fox, Jon E., und Cynthia Miller-Idriss. 2008. Everyday nationhood. Ethnicities 8(4):536-563.

Fukuyama, Francis. 2018. Identity: the demand for dignity and the politics of resentment. New York: Farrar Straus and Giroux.

Geist, Michael. 2004. Governments and country-code top level domains: a global survey. Version 2.0. https://www.itu.int/osg/spu/forum/intgov04/contributions/governmentsandcctldsfeb04.pdf. Zugegriffen: 23. Sept. 2018.

Gellner, Ernest. 1999. Nationalismus: Kultur und Macht. Berlin: Siedler.

Gerrand, Peter. 2006. Cultural diversity in cyberspace: the Catalan campaign to win the new .cat top level domain. First Monday https://doi.org/10.5210/fm.v11i1.1305.

Glen, Carol M. 2014. Internet governance: territorializing cyberspace? Politics \& Policy 42(5):635-657.

Goldsmith, Jack L., und Tim Wu. 2006. Who controls the Internet? Illusions of a borderless world. New York: Oxford University Press.

Goldsmith, Jack, und Stuart Russell. 2018. Strengths become vulnerabilities: how a digital world disadvantages the United States in its international relations. https://www.hoover.org/sites/default/files/ research/docs/381100534-strengths-become-vulnerabilities.pdf. Zugegriffen: 28. Mai 2020.

Habermas, Jürgen. 1998a. Die postnationale Konstellation: Politische Essays. Frankfurt am Main: Suhrkamp.

Habermas, Jürgen. 1998b. Volkssouveränität als Verfahren. In Faktizität und Geltung Beiträge zur Diskurstheorie des Rechts und des demokratischen Rechtsstaats, Hrsg. Jürgen Habermas, 600-631. Frankfurt am Main: Suhrkamp.

Hathaway, Melissa E. 2014. Connected choices: how the Internet is challenging sovereign decisions. American Foreign Policy Interests 36(5):300-313.

Hill, Jonah Force. 2012. Internet fragmentation: highlighting the major technical, governance and diplomatic challenges for U.S. policy makers. Research Paper. Cambridge, MA: John F. Kennedy School of Government.

Hobsbawm, Eric J. 2005. Nationen und Nationalismus: Mythos und Realität seit 1780. Frankfurt am Main: Campus.

Hrynyshyn, Derek. 2008. Globalization, nationality and commodification: the politics of the social construction of the internet. New Media \& Society 10(5):751-770.

ICANN/GAC. 2000. Principles and guidelines for the delegation and administration of country code top level domains. https://gac.icann.org/principles-and-guidelines/public/principles-cctlds.pdf. Zugegriffen: 27. Febr. 2021. 
Jamieson, Kathleen Hall. 2018. Cyberwar: how Russian hackers and trolls helped elect a president; what we don't, can't, and do know. New York: Oxford University Press.

Johnson, David R., und David Post. 1996. Law and borders: the rise of law in cyberspace. Stanford Law Review 48(5):1367.

Kedourie, Elie. 1971. Nationalismus. München: List.

Keller, Reiner. 2008. Wissenssoziologische Diskursanalyse. Grundlegung eines Forschungsprogramms. Wiesbaden: VS

Kohn, Hans. 1955. Nationalism: its meaning and history. Princeton: Van Nordstrand Company.

Lambach, Daniel. 2020. The territorialization of cyberspace. International Studies Review 22(3):482-506.

Lanoszka, Alexander. 2019. Disinformation in international politics. European Journal of International Security 4(2):227-248.

Macron, Emmanuel. 2018. Déclaration de M. Emmanuel Macron, Président de la République, sur la liberté de la presse, la lutte contre les fausses nouvelles, l'économie des médias et sur la relation entre les gouvernants et les médias, à Paris le 3 janvier 2018. https:/www.vie-publique.fr/discours/ 204657-declaration-de-m-emmanuel-macron-president-de-la-republique-sur-la-li. Zugegriffen: 26. März 2020.

Meinrath, Sascha. 2013. We can't let the Internet become balkanized. http://www.slate.com/articles/ technology/future_tense/2013/10/internet_balkanization_may_be_a_side_effect_of_the_snowden_ surveillance.html. Zugegriffen: 20. Juni 2017.

Möllers, Norma. 2021. Making digital territory: cybersecurity, techno-nationalism, and the moral boundaries of the state. Science, Technology, \& Human Values 46(1):112-138.

Mueller, Milton. 2017. Will the Internet fragment? Surveillance, cybersecurity and Internet governance. Cambridge: Polity Press.

Mueller, Milton L., und Farzaneh Badiei. 2017. Governing Internet territory: ICANN, sovereignty claims, property rights and country code top-level domains. The Columbia Science \& Technology Law Review XVIII:436-491.

Nissenbaum, Helen. 2005. Where computer security meets national security. Ethics and Information Technology 7(2):61-73.

O'Neil, Cathy. 2017. Weapons of math destruction: how big data increases inequality and threatens democracy. New York: B/D/W/Y Broadway Books.

Pfetsch, Barbara, Annett Heft, und Curd Knüpfer. 2019. Transnationale Öffentlichkeiten in der Digitalen Gesellschaft: Konzepte und Forschungsperspektiven. In Politik in der digitalen Gesellschaft. Zentrale Problemfelder und Forschungsperspektiven, Hrsg. Jeanette Hofmann, Norbert Kersting, Claudia Ritzi, und Wolf J. Schünemann, 83-101. Bielefeld, Berlin: transcript; Walter de Gruyter.

Pistor, Katharina. 2020. Statehood in the digital age. Constellations 27(1):3-18.

Pohle, Julia, und Thorsten Thiel. 2019. Digitale Vernetzung und Souveränität: Genealogie eines Spannungsverhältnisses. In Internet und Staat. Perspektiven auf eine komplizierte Beziehung, Hrsg. Isabelle Borucki, Wolf J. Schünemann, 57-80. Baden-Baden: Nomos.

Pohle, Julia, und Thorsten Thiel. 2020. Digital sovereignty. Internet Policy Review https://doi.org/10. $14763 / 2020.4 .1532$.

Postel, Jon, und J. Reynolds. 1984. Domain requirements: request for comments (920)

Pries, Ludger. 2010. Transnationalisierung: Theorie und Empirie grenzüberschreitender Vergesellschaftung. Wiesbaden: VS.

Randazza, Marc. 2016. Kosovo's digital independence: time for Kosovo's CCTLD. Wisconsin International Law Journal 33(4):667-700.

Rid, Thomas. 2018. Mythos Cyberwar: Über digitale Spionage, Sabotage und andere Gefahren. Hamburg: Edition Körber.

Rid, Thomas. 2020. Active measures: the secret history of disinformation and political warfare. London: Profile Books.

Ritzi, Claudia, und Alexandra Zierold. 2019. Souveränität unter den Bedingungen der Digitalisierung. In Internet und Staat. Perspektiven auf eine komplizierte Beziehung, Hrsg. Isabelle Borucki, Wolf J. Schünemann, 37-59. Baden-Baden: Nomos.

Sassen, Saskia. 2008. Das Paradox des Nationalen: Territorium, Autorität und Rechte im globalen Zeitalter. Frankfurt, M.: Suhrkamp.

Schünemann, Wolf J. 2014. Subversive Souveräne: Vergleichende Diskursanalyse der gescheiterten Referenden im europäischen Verfassungsprozess. Wiesbaden: Springer VS.

Schünemann, Wolf J. 2020. Cybersicherheit. In Handbuch Digitalisierung in Staat und Verwaltung, Hrsg. Tanja Klenk, Frank Nullmeier, und Göttrik Wewer, 1-11. Wiesbaden: Springer. 
Schünemann, Wolf J., und Reiner Keller. 2016. Narrativer Nationalismus: Die Wissenssoziologische Diskursanalyse zur Untersuchung kultureller Kontexte der politischen Auseinandersetzung in Europa. In Die andere Seite der Politik. Theorien kultureller Konstruktion des Politischen, Hrsg. Wilhelm Hofmann, Renate Martinsen, 55-83.

Schütz, Alfred. 1993. Der Sinnhafte Aufbau der sozialen Welt: Eine Einleitung in die verstehende Soziologie. Frankfurt am Main: Suhrkamp.

Shackelford, Scott, Bruce Schneier, Michael Sulmeyer, Anne Boustead, Ben Buchanan, Amanda Craig, Trey Herr, Malekos Smith, und J. Zhanna. 2016. Making democracy harder to hack: should elections be classified as 'critical infrastructure?'. University of Michigan Journal of Law Reform 50(3):629-668.

Smith, Anthony David. 2014. Nationalism: theory, ideology, history. Cambridge: Polity Press.

Thiel, Thorsten. 2019. Souveränität: Dynamisierung und Kontestation in der digitalen Konstellation. In Politik in der digitalen Gesellschaft. Zentrale Problemfelder und Forschungsperspektiven, Hrsg. Jeanette Hofmann, Norbert Kersting, Claudia Ritzi, und Wolf J. Schünemann, 47-60. Bielefeld, Berlin: transcript; Walter de Gruyter.

Thornton, Rod, und Marina Miron. 2019. Deterring Russian cyber warfare: the practical, legal and ethical constraints faced by the United Kingdom. Journal of Cyber Policy 4(2):257-274.

Tönnies, Ferdinand. 1887. Gemeinschaft und Gesellschaft: Abhandlung des Communismus und des Socialismus als empirischer Culturformen. Berlin: Fues.

Vick, Douglas W. 2001. Exporting the first amendment to cyberspace: the Internet and state sovereignty. In Media and globalization. Why the state matters, Hrsg. Nancy Morris, Silvio Ricardo Waisbord, und Kaarle Nordenstreng, 3-19. Lanham: Rowman \& Littlefield.

Werron, Tobias. 2018. Der globale Nationalismus. Berlin: Nicolai.

Wu, Timothy S. 1997. Cyberspace sovereignty? The Internet and the international system. jolt.law.harvard.edu/ articles/pdf/v10/10HarvJLTech647.pdf. Zugegriffen: 15. Jan. 2015. 